\title{
PUBLICATIONS
}

\section{SOFTWARE FOR SCIENTISTS}

Chemintosh Desk Accessory 1.42. Available from SoftShell International, 2004 North 12th St., Grand Junction, CO 81501. \$295 (20\% academic discount).

Chemintosh is a drawing program that anyone who creates chemical structures will greatly appreciate. As a desk accessory (DA), it is available from within other programs, such as word processors and spreadsheets. This is a considerable advantage for those not using Multifinder, since you do not need to quit your application in order to draw chemical structures. Furthermore, Chemintosh chemical structures can be combined with other drawing programs to produce complex figures for manuscripts.

Like other drawing programs, Chemintosh has a palette of graphic tools. However, these tools are specific for structural drawing, such as for creating three to seven carbon rings, benzene, or chair and boat configurations. Other tools draw bonds, such as wedged, hashed wedged, single, double, triple, as well as arrows for reactions, equilibrium, resonance, and movement of electrons. These tools are quite flexible and can be edited to provide desired lengths and angles and to change standard parameters. The program is intuitive and can be used initially without even opening the manual, although the function of some of the tools is not immediately obvious and requires consulting the manual.

The best way to evaluate the program is to look at some examples:

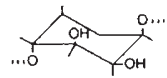

$\alpha$-L-Gulopyranosyluronic acid unit

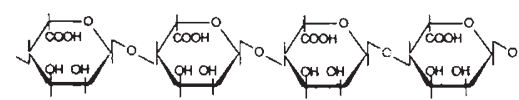

Guluronic acid polymer segment of alginic acid

by Keith Redenbaugh, principal scientist, Calgene, 1920 Fifth Street, Davis, CA 95616.
There was a problem with printing within Chemintosh while running $\mathrm{Mi}$ crosoft Word 4.0: Printing was not possible even with all other windows closed. This is likely an incompatibility with Word 4.0. Also, when using Geneva 12 point font, the letters ran together (Helvetica did not have this problem).

The program has a powerful method for creating complex chemical strings, but requires careful consultation with the manual. Many other powerful and useful features also require thorough reading. The manual contains an excellent six-page reference section that lists functions of all the tools with appropriate results using command keys. Complete explanations of all the functions follow a condensed 16-page overview. Becoming proficient with Chemintosh takes time, but the result is the ability to rapidly create and modify chemical structures.

The vendor will supply free the Chemintosh Molecular Mass Calculator, a simple DA for calculating the molecular mass of any chemical formula: The formula is entered without spaces, but with parentheses as needed. Press the return key to obtain an instant mass reading with five significant numbers.

Suitcase II. Available from Fifth Generation Systems, 11200 Industriplex Blvd., Baton Rouge, LA 70909. \$79. Hardware requirements: Macintosh 512KE, Plus, SE, Mac II, or Mac IIcx with System 4.1 or later. Not available for MS-DOS.

$T$ he other software to consider is not directly used in writing or scientific research, but it does make possible the use of larger numbers of desk accessories (DAs) and fonts than allowed by the Macintosh system. For example, my Mac I has 29 desk accessories and 40 fonts. Although I use many only sparingly, I do use 18 of the DAs and six of the fonts routinely. So a utility that expands beyond the 15-DA limit of the Mac operating system is essential. For anyone who finds DAs such as EndNote and Chemintosh useful, that limitation will quickly hinder your flexibility and productivity.

Suitcase II is a startup utility (INIT) that loads automatically into a system and provides an improved method for handling classes of resources. With Suitcase, virtually unlimited numbers of desk accessories under the Apple menu can be customized, such as putting the most frequently used ones on top. The key to using Suitcase is to remember to place all your DAs in one or a few "suitcases" (files). Because the system allows only 12 suitcases, you need to pack DAs, fonts, function keys, and beep sounds into these suitcases. While a bit confusing at first-particularly if you have relied on Apple's Font/DA mover-it quickly becomes apparent how powerful and useful the suitcases are.

Fonts have less of a numbers limitation, but are still handled in the same fashion. Of great value is that fonts can be displayed in their own typefaces, either through the Suitcase accessory or directly under the font menu of your word processor or other application. The latter is easily done by holding down the option key when pulling down the font menu. This saves considerable time over using the standard Mac system Key Caps DA.

Another feature is a font and sound compression utility that saves up to 60 percent of disk space and automatically unpacks them when used. Once you install this, you will not even notice it is in your system. As your library of fonts and sounds increases, this compression utility becomes essential.

Using Suitcase is a bit more complicated than it should be, although there are only a few hierarchial windows and choices to make. Part of this confusion would be alleviated if you could load DAs, fonts, etc. into files within the utility and not have to rely on Font/DA Mover. If not careful, you will end up opening individual DAs as well as suitcases and reach the 12 limit quickly. While confusing at first, the excellent manual explains this well.

For a free copy of this article (while available), write in 506 on Reader Service Card 